\title{
Rassegna
}

\section{Acquaretici: dalla fisiologia alla clinica}

\author{
P. Lombari, C. Altobelli, F. Trepiccione, G. Capasso
}

Cattedra di Nefrologia, Dipartimento di Medicina Interna, Seconda Università di Napoli, Napoli

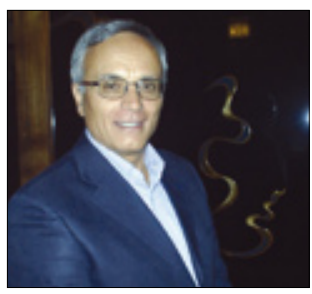

Giovambattista Capasso

\section{Le Acquaporine}

Il rene svolge un ruolo chiave nel bilancio idro-salino regolando la quantità di acqua e soluti da riassorbire in base alle esigenze dell'organismo. Il riassorbimento di acqua avviene in maniera costitutiva lungo i segmenti del nefrone detti "leaky", cioè costitutivamente permeabili all'acqua. Lungo il dotto collettore, invece, avviene una più fine regolazione della quota d'acqua riassorbibile. Il dotto collettore infatti ha una permeabilità variabile in dipendenza dai livelli circolanti di ADH (ormone antidiuretico). Il gradiente osmotico tra il lume tubulare e l'interstizio midollare sostiene il riassorbimento netto di acqua.

Per lungo tempo si è pensato che, essendo l'acqua una molecola molto piccola, ubiquitaria, potesse essere riassorbita liberamente in qualsiasi punto della membrana plasmatica. Nel 1993 Peter Agre e collaboratori identificarono per la prima volta negli eritrociti la presenza di canali di membrana selettivi per l'acqua, le acquaporine (AQP) (1). Grazie a studi successivi riuscirono a dimostrare l'esistenza di questi canali sulla membrana plasmatica di altri tipi cellulari. Attualmente sono state identificate 13 isoforme di AQP. A livello renale ne sono presenti solo alcune, l'AQP-1 nel tubulo prossimale e nel tratto discendente sottile dell'ansa di Henle (segmento leaky) che riassorbe il 90\% dell'acqua filtrata, l'AQP-7 nel tubulo prossimale, nonché le AQP-2 -3 e -4 nel dotto collettore. L'AQP-2 è espressa sul versante apicale delle cellule principali del dotto collettore, mentre l'AQP-3 e l'AQP-4 sul versante basolaterale delle stesse cellule. Il sistema delle AQP-2 e AQP-3-4, regolato dall'ADH, è responsabile della regolazione fine del riassorbimento dell'acqua nel dotto collettore. Alcune evidenze recenti individuerebbero l'AQP-5 lungo il segmento distale del nefrone, la cui funzione non è ancora nota. (Fig. 1) . L'AQP-2 è sintetizzata nelle cellu- le principali del tubulo collettore ma la quantità di canali attivi, cioè trasferiti dalle vescicole citoplasmatiche sulla membrana apicale, è sensibilmente regolata dall'ormone $\mathrm{ADH}$ (2). In risposta a piccoli incrementi dell'osmolalità plasmatica, alla riduzione del volume plasmatico circolante, all'attivazione del sistema simpatico, alla nausea, al vomito e al freddo, l'ADH viene rilasciato dalla neuroipofisi promuovendo il riassorbimento di acqua nel dotto collettore renale e di sodio nel tratto ascendente spesso dell'ansa di Henle. L'ADH agisce legandosi al recettore di tipo $\mathrm{V}_{2}$ sulla membrana basolaterale delle cellule principali del dotto collettore. Da qui, viene attivata l'adenilato ciclasi con conseguente produzione dell'AMP ciclico (cAMP), secondo messaggero per le protein chinasi A (PKA). Le PKA fosforilano le molecole di AQP-2 presenti nelle vescicole citoplasmatiche, consentendo la fusione delle vescicole con la membrana apicale (Fig. 2).

\section{Ruolo dell'ADH e dell'AQP-2 nelle patologie associate a disordini dell'omeostasi idrica}

Un'alterazione della fisiologia dell'AQP-2 incorre nei disordini dell'omeostasi dell'acqua. Le condizioni cliniche associate a iperstimolazione dell'asse ADH-AQP2 (scompenso cardiaco congestizio, cirrosi epatica e sindrome da inappropriata secrezione di $\mathrm{ADH}$ ) (SIADH) (3), sono accomunate da iponatriemia proprio in virtù di un disequilibrio dell'omeostasi idrica piuttosto che ad alterazioni del bilancio del sodio. Qui focalizzeremo la nostra attenzione sul disequilibrio dell'omeostasi idrica in corso di SIADH, cirrosi epatica e scompenso cardiaco.

La SIADH è una sindrome associata a una secrezione inappropriata, ipotalamica o ectopica, di $\mathrm{ADH}$. In corso di SIADH la secrezione dell'ADH è inappropriata rispetto all'osmolalità plasmatica, infatti, l'iposmolalità plasmatica non è in grado di inibirne la secrezione. Questa 


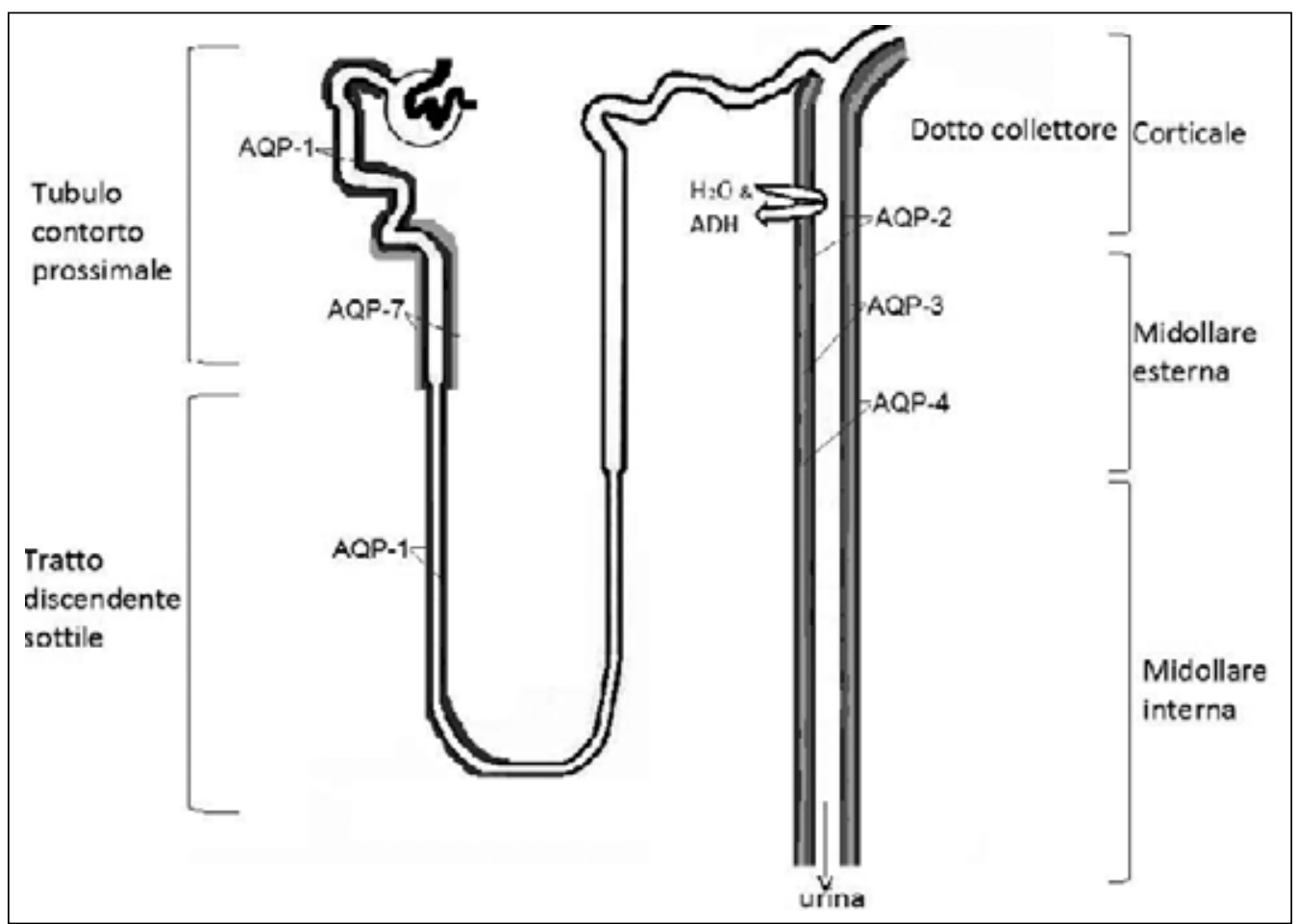

Fig. 1 - Espressione delle Acquaporine lungo il nefrone. Precisamente l'AQP-1 è localizzata lungo il tubulo contorto prossimale e tratto discendente sottile dell'ansa di Henle (segmenti leaky, costitutivamente permeabile all'acqua) mentre l'AQP-7 nel tratto sp3 del tubulo contorto prossimale. Il sistema delle acquaporine regolato dall'ADH è espresso nel dotto collettore: AQP-2 versante apicale delle cellule principali, AQP-3 e 4 versante basolaterale delle stesse cellule.

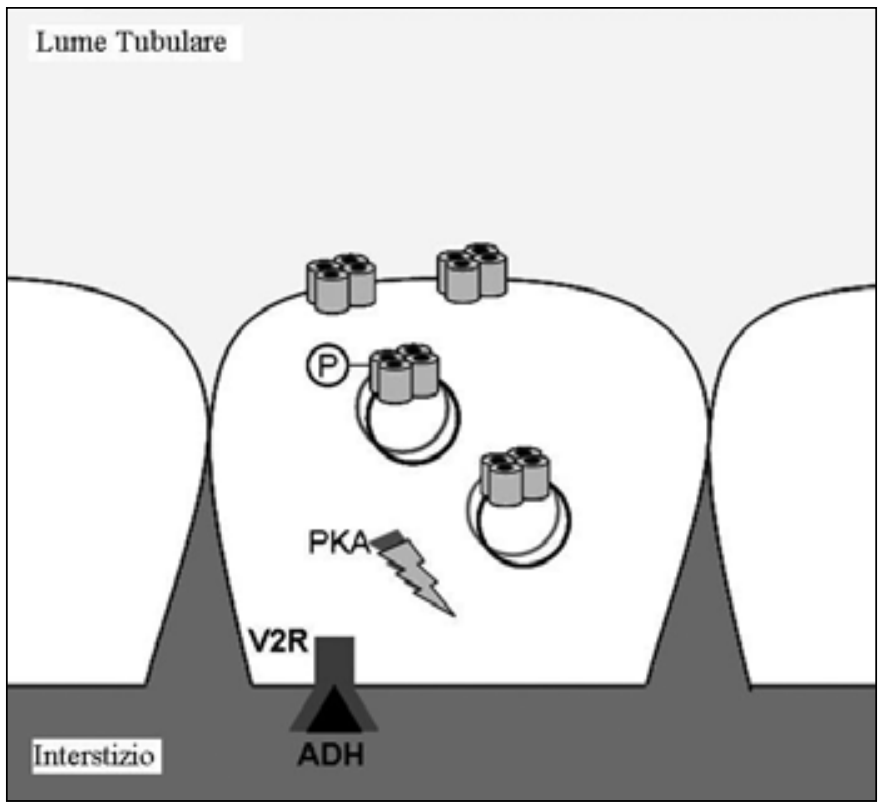

Fig. 2 - L'interazione tra l'ADH e il proprio recettore di tipo $V_{2}$ (basolaterale) promuove una cascata di eventi intracitoplasmatici responsabile dell'espressione dell'AQP-2 sul versante apicale della cellula principale del dotto collettore.

patologia può manifestarsi in corso di disturbi del SNC, di patologie infettive (soprattutto polmoniti), autoimmunitarie, neoplastiche (microcitoma polmonare), a seguito di interventi chirurgici (soprattutto di neurochirur- gia) o assunzione di farmaci (antiepilettici, inibitori del reuptake della serotonina ecc). L'aumentata secrezione dell'ADH induce una maggiore espressione delle AQP2 lungo il dotto collettore. Da qui un riassorbimento di acqua disgiunto dalle reali esigenze dell'omeostasi idrica e l'instaurarsi dell'iponatriemia. Elementi diagnostici di questa condizione sono l'iponatremia associata a iposmolalità plasmatica (cut-off $<275 \mathrm{mOsm} / \mathrm{kg} \mathrm{H}_{2} \mathrm{O}$ ), a un'osmolalità urinaria maggiore di $100 \mathrm{mOsm} / \mathrm{kg} \mathrm{H}_{2} \mathrm{O}$, a sodiuria $>40 \mathrm{mEq} / \mathrm{L}$, riduzione dell'uricemia in soggetti con funzionalità renale, cardiaca, epatica, surrenalica e tiroidea normale. Non è ancora chiaro quale sia il grado di insufficienza renale in cui questa condizione può ancora determinarsi. Le manifestazioni cliniche dell'iponatriemia dipendono dalla gravità e dalla velocità con cui essa si instaura. Il trattamento della SIADH è duplice, in primo luogo bisogna ripristinare la natremia fisiologica (in caso si sospetti un'iponatremia acuta la correzione dovrebbe essere apportata in 2 giorni) e inoltre è necessario, laddove possibile, trattare la causa scatenante. Ad oggi, il trattamento dell'iponatriemia in corso di SIADH (4) prevede innanzitutto la restrizione idrica (disponibile, economico, ma scarsamente compliante per i pazienti). A seconda delle necessità è possibile ricorrere a soluzioni saline ipertoniche al $3 \%$, diuretici dell'ansa e altri farmaci (la demeclociclina, l'urea ed il litio). Da qualche mese in Italia, il trattamento delliponatriemia da SIADH si avvale dei farmaci antagonisti non peptidici del recettore $\mathrm{V}_{2}$ dell'ADH. 
Nella cirrosi epatica la vasodilatazione splancnica si associa a una riduzione del volume plasmatico effettivo, stimolando i barorecettori carotidei e renali (5) al riassorbimento del sodio e all'incremento della secrezione dell'ADH (6). L'elevata secrezione dell'ADH è responsabile di una notevole espressione di AQP-2, accentuando la permeabilità del dotto collettore. Uno studio di Ivansen e collaboratori (7), ha evidenziato un'escrezione urinaria dell'AQP-2 otto volte maggiore nei pazienti cirrotici rispetto ai soggetti sani. Nella cirrosi epatica la clearance dell'acqua libera è conservata inizialmente, e si riduce con la progressione della malattia, determinando un'importante ritenzione idrica responsabile dell'ascite e dell'iponatriemia da diluizione. L'iponatriemia nei cirrotici non dipende dall'assunzione dei diuretici, ma può essere aggravata della loro somministrazione.

Nello scompenso cardiaco congestizio l'ipoperfusione degli organi e la riduzione della pressione arteriosa, dipendenti dal deficit di pompa, stimolano i barorecettori carotidei e renali con conseguente secrezione di renina, $\mathrm{ADH}$ e norepinefrina. L'attivazione di questi meccanismi di compenso promuove un notevole riassorbimento di sodio e acqua mediati dall'ADH. Dallo studio di $\mathrm{Xu}$ e collaboratori, condotto su un modello animale di scompenso cardiaco, è emersa un'aumentata sintesi di $\mathrm{ADH}$ e $\mathrm{AQP}-2$, tale incremento è precoce e può essere inibito, dalla somministrazione dei farmaci antagonisti non peptidici del recettore $\mathrm{V}_{2}$ dell'ADH (10). Lo studio condotto da Funayama su un gruppo di pazienti affetti da scompenso cardiaco ha confermato l'elevata escrezione urinaria delle AQP-2 nei pazienti in III e IV classe NYHA. Nello scompenso cardiaco congestizio c'è quindi una stretta correlazione tra la gravità della patologia e i livelli plasmatici di $\mathrm{ADH}$, nonché tra i livelli plasmatici dell'ADH e l'escrezione urinaria di AQP-2 (11). Una conseguenza importante della ritenzione massiva di acqua è l'iponatriemia da diluizione, comunemente riscontrata in questi pazienti.

Liponatremia caratterizzante tutte e tre queste condizioni è nella pratica clinica spesso considerata un nonproblema, ma è uno degli elementi sintomatologici più significativi e associati a una maggiore mortalità (12).

\section{Vaptani: farmaci antagonisti non peptidici del recettore dell'ADH}

I Vaptani sono antagonisti non peptidici del recettore dell'ADH (13). Tre sono i sottotipi dei recettori dell'ADH: $\mathrm{V}_{1 \mathrm{a}}, \mathrm{V}_{1 \mathrm{~b}}, \mathrm{e} \mathrm{V}_{2}$. In questa trattazione ci soffermeremo sui farmaci antagonisti del recettore $\mathrm{V}_{2}$. Il recettore $\mathrm{V}_{2}$ è espresso sul versante basolaterale delle cellule principa- li del dotto collettore renale e la sua stimolazione comporta un aumentato riassorbimento di acqua. L'azione antagonizzante i recettori $\mathrm{V}_{2}$ dell'ADH fa si che questi farmaci vengano più comunemente conosciuti come acquaretici perché, a differenza dei diuretici, sono in grado di indurre una diuresi altamente ipotonica senza alterare l'escrezione degli elettroliti.

\section{Il Tolvaptan}

Il Tolvaptan, un Vaptano specifico per i recettori $\mathrm{V}_{2}$, attivo per via orale, è stato utilizzato per gli studi SALT1 e SALT2, in pazienti con iponatriemia $(<135 \mathrm{mmol} / \mathrm{L})$ associata a scompenso cardiaco, cirrosi epatica e sindrome da inappropriata secrezione di ormone antidiuretico, per valutarne la reversibilità degli effetti e la sicurezza del farmaco. I trials SALT1 e SALT2 sono studi multicentrici condotti in doppio cieco e randomizzati, Tolvaptan verso placebo. In questi studi la somministrazione del Tolvaptan è stata effettuata per 30 giorni, sono stati valutati, tra l'altro, l'incremento della sodiemia al giorno $4 \mathrm{e}$ al giorno 30 (end points primari) e l'incremento del sodio sierico in corso di iposodiemia marcata $(<130 \mathrm{mEq} / \mathrm{L}) \mathrm{e}$ moderata $(\approx 130-135 \mathrm{mEq} / \mathrm{L})$ (end points secondari). Da queste analisi è risultato un significativo aumento della sodiemia nei pazienti trattati con Tolvaptan rispetto al gruppo placebo (14). Lo studio è proseguito con la valutazione degli effetti della somministrazione di Tolvaptan a lungo termine (4 anni) sugli stessi pazienti utilizzati negli studi SALT1 e SALT2, il farmaco è stato somministrato anche ai pazienti che nel precedente studio erano stati assegnati al gruppo placebo (trial SALTWATER). In sintesi dai tre studi è risultato che Tolvaptan è efficace nella terapia dell'iponatremia eu/ipervolemica, ha una relativa sicurezza per eventi avversi maggiori a 4 anni, gli effetti collaterali più comuni sono pollachiuria, sete, stanchezza, bocca secca, polidipsia e poliuria (15).

Oltre agli effetti in corso di iponatriemia, il Tolvaptan è stato utilizzato in altri trials ad esempio negli Studi Everest e Meteor. Nello Studio Everest pazienti ospedalizzati affetti da scompenso cardiaco acuto sono stati trattati, entro 48 ore dalla comparsa della sintomatologia, con Tolvaptan al fine di stimarne gli effetti sulla mortalità e morbilità a breve e a lungo termine. Nessuna variazione in termini di mortalità è stata riportata in corso di insufficienza cardiaca congestizia normo e iponatremica (16). Dallo Studio Meteor non è emersa alcuna azione efficace del farmaco sul rimodellamento del ventricolo sinistro in pazienti con insufficienza cardiaca congestizia dopo un anno di trattamento (17).

$\mathrm{E}$ attualmente in sperimentazione l'impiego del Tolvap- 
tan per ritardare la progressione dell'insufficienza renale nella malattia renale policistica autosomica dominante (ADPDK) (studio Tempo). Lo studio Tempo è un trial clinico in fase 3 , multicentrico, in doppio cieco, controllato Tolvaptan vs placebo (2:1) e a bracci paralleli, della durata di tre anni, e ha lo scopo di determinare l'efficacia e la sicurezza a lungo termine del Tolvaptan in soggetti adulti affetti da ADPKD. Questa patologia è caratterizzata dalla formazione e dal successivo ingrandimento di cisti renali responsabili della progressiva riduzione della funzionalità renale. Nei pazienti affetti da malattia renale policistica sono stati riscontrati elevati livelli di $\mathrm{ADH}$. L'ADH legandosi al proprio recettore $\mathrm{V}_{2}$ induce un aumento della sintesi dell'cAMP, il quale è responsabile dell'attivazione di meccanismi di trasduzione intracitoplasmatici, tra cui la cascata ERK. Tra l'altro, pare che l'attivazione di questa cascata sia indispensabile per promuovere la proliferazione cellulare e lo sviluppo delle cisti in questi pazienti. Un dato che avvalora l'importanza cruciale dell'ADH nella crescita delle cisti è che esse si organizzano soprattutto a livello del dotto collettore là dove sono localizzati i recettori dell'ADH. In corso di APDKD i livelli di cAMP sono ulteriormente incrementati dall'aumento dei livelli di calcio citoplasmatico dipendenti dalle alterazioni genetiche tipiche di questa patologia (18). L'uso del Tolvaptan nei pazienti con $\mathrm{ADPKD}$ ha come razionale l'antagonizzazione di questo meccanismo.

\section{Conclusioni}

L'iponatremia è spesso espressione di un disequilibrio dell'omeostasi dell'acqua corporea. Sebbene spesso misdiagnosticata, l'iponatremia correla con una più alta mortalità, per qualsivoglia malattia di base. I Vaptani, farmaci antagonisti del recettore dell'ADH, stanno diventando sempre più importanti per il trattamento dell'iponatriemia eu/ipervolemica. La SIADH oggi e nel prossimo futuro la cirrosi epatica, lo scompenso cardiaco, la malattia renale policistica sono condizioni in cui l'utilizzo degli acquaretici potrebbe essere un ulteriore strumento nelle mani del nefrologo. Le nuove evidenze fisiopatologiche sul meccanismo della concentrazione urinaria e i disordini ad esso correlati hanno fornito al clinico nuovi target terapeutici, realizzando così un ideale ponte tra studi sperimentali e applicazione clinica.

\author{
Indirizzo degli Autori: \\ Prof. Giovambattista Capasso \\ Cattedra di Nefrologia \\ Padiglione 17 Policlinico Nuovo \\ Via Pansini 5 \\ 80131 Napoli \\ gb.capasso@unina2.it
}

\section{Bibliografia}

1. Agre P, Preston GM, Smith BL, Jung JS, Raina S, Moon C, Guggino WB, Nielsen S. Aquaporin CHIP: The archetypal molecular water channel. Am J Physiol 1993; 265: F463F476.

2. Nielsen S, Digiovanni SR, Christensen EI, Knepper MA, Harris HW. Cellular and subcellular immunolocalization of vasopressin regulated water channel in rat kidney. Proc Natl Acad Sci USA 1993; 90: 11663-7.

3. Nielsen S, Kwon TH, Christensen BM, Promeneur D, Frokiaer J, Marples D. Physiology and pathophysiology of renal aquaporins. J Am Soc Nephrol 1999; 10: 647-63.

4. Sherlock M, Thompson CJ. The syndrome of inappropriate antidiuretic hormone: current and future management options. Eur J Endocrinol 2010; 162(Suppl 1): S13-8.
5. Schrier RW, Arroyo V, Bernardi M, Epstein M, Henricksen J, Rodes J. Peripheral arterial vasodilation hypothesis: A proposal for the initiation of renal sodium and water retention in cirrhosis. Hepatology 1988; 8: 1151-7.

6. Kim J, Summer S, Howard R, Schrier RW. Vasopressin gene expression in rats with experimental cirrhosis. Hepatology 1993; 17: 143-7.

7. Ivarsen P, Frokiaer J, Aagaard N, Hansen E, Bendtsen F, Nielsen S, Vilstrup H. Increased urinary excretion of aquaporin 2 in patients with liver cirrhosis. Gut 2003; 52: 1194-9.

8. Kim JK, Michel JB, Soubrier F, DurrJ, Corvol P, Schrier RW. Arginine vasopressin gene expression in chronic cardiac failure in rats. Kidney Int 1990 Nov; 38(5): 81822.

9. Goldsmith SR, Francis GS, Cowley AW, Jr. Arginine vaso- 
pressin and the renal response to water loading in congestive heart failure. Am J Cardiol 1986 Aug 1; 58(3): 295-9.

10. Xu D, Martin PY, Ohara M, et al. Upregulation of aquaporin-2 water channel expression in chronic heart failure. J Clin Invest 1997; 99: 1500-05.

11. Funayama H, Nakamura T, Saito T, et al. Urinary excretion of aquaporin-2 water channel exaggerated dependent upon vasopressin in congestive heart failure. Kidney Int 2004 Oct; 66(4): 1387-92.

12. Packer M, Lee WH, Kessler PD. Preservation of glomerular filtration rate in human heart failure by activation of the renin-angiotensin system. Circulation 1986 Oct; 74(4): 766-74.

13. Decaux G, Soupart A, Vassart G. Non-peptide argininevasopressin antagonists: the vaptans. Lancet 2008 May 10; 371(9624): 1624-32.

14. Schrier RW, Gross P, Gheorghiade M, Berl T, Verbalis JG, Czerwiec FS, Orlandi C; SALT Investigators. Tolvaptan, a selective oral vasopressin V2-receptor antagonist, for hyponatriemia. N Engl J Med 2006 Nov 16; 355(20): 2099112.

15. Berl T, Quittnat-Pelletier F, Verbalis JG, Schrier RW, Bichet DG, Ouyang J, Czerwiec FS; SALTWATER Investigators. Oral tolvaptan is safe and effective in chronic hyponatriemia. J Am Soc Nephrol 2010 Apr; 21(4):705-12.

16. Konstam MA, Gheorghiade M, Burnett JC, Jr., et al. Effects of oral tolvaptan in patients hospitalized for worsening heart failure: The EVEREST Outcome Trial. JAMA 2007 Mar 28; 297(12):1319-31.

17. Udelson JE, McGrew FA, Flores E, et al. A Multicenter, randomized, placebo-controlled study on the effects of oral tolvaptan on left ventricular dilatation and function in patients with heart failure and systolic dysfunction. J Am Coll Cardiol 2007 Jun 5; 49(22): 2151-9.

18. Torres VE, Wang X, Qian Q, Somlo S, Harris PC, Gattone VH 2nd. Effective treatment of an orthologous model of autosomal dominant polycystic kidney disease. Nat Med 2004; 10: 363-4. 